Cahiers $d u$ MONDE RUSSE

\section{Cahiers du monde russe}

Russie - Empire russe - Union soviétique et États indépendants

$48 / 4 \mid 2007$

Varia

\title{
Zoltan Barany, Democratic Breakdown and the Decline of the Russian Military
}

\author{
Elisabeth Sieca-Kozlowski
}

\section{OpenEdition \\ Journals}

Édition électronique

URL : https://journals.openedition.org/monderusse/6105

DOI : 10.4000/monderusse.6105

ISSN : $1777-5388$

Éditeur

Éditions de l'EHESS

Édition imprimée

Date de publication : 2 décembre 2007

Pagination : 781-784

ISBN : 978-2-7132-2148-4

ISSN : $1252-6576$

Référence électronique

Elisabeth Sieca-Kozlowski, «Zoltan Barany, Democratic Breakdown and the Decline of the Russian Military », Cahiers du monde russe [En ligne], 48/4 | 2007, mis en ligne le 16 juin 2009, consulté le 04 septembre 2022. URL : http://journals.openedition.org/monderusse/6105 ; DOI : https://doi.org/ 10.4000/monderusse.6105

Ce document a été généré automatiquement le 4 septembre 2022

Tous droits réservés 


\title{
Zoltan Barany, Democratic Breakdown and the Decline of the Russian Military
}

\author{
Elisabeth Sieca-Kozlowski
}

\section{RÉFÉRENCE}

Zoltan BARANY, Democratic Breakdown and the Decline of the Russian Military.

Princeton : Princeton University Press, 2007, 264 p.

1 Le livre de Zoltan Barany tente d'expliquer les raisons du déclin de l'armée russe et son incapacité à se réformer à travers l'analyse de l'évolution des relations civilo-militaires, de Gorbačev à Putin. Considérées comme mesure de démocratisation, ces relations (et leur décryptage) doivent permettre de mettre en lumière l'orientation du pays dans cette voie ou dans celle de l'autoritarisme. L'évolution des rapports entre l'institution présidentielle et les forces armées donne des éléments de réponse à cette question et explique l'absence de réformes militaires radicales en Russie.

La détérioration de l'armée s'amorce au début des années 1980, s'amplifie avec Gorbačev, pour culminer avec El'cin. Bien qu'il prenne acte d'une amélioration des conditions de vie des militaires et de la prise de conscience de la nécessité d'une réforme avec Putin, Barany estime que les causes de la situation difficile des forces armées n'ont été ni analysées ni éliminées : l'armée n'a pas fait l'objet de réformes suffisamment profondes. Ce qui a été fait a été mal fait, parfois d'une façon laissant penser que l'on cherchait à recréer... l'Armée rouge. Une armée russe menaçante peut-elle émerger à nouveau dans un proche avenir? C'est une des questions qui sous-tend cet ouvrage. Dans le contexte de retour sur la scène internationale d'une Russie réaffirmant son ambition de puissance et de grandeur, les États-Unis doivent-ils s'inquiéter? Ce livre entend donc présenter l'évolution des relations civilo-militaires à partir de concepts de la science politique et servir par là de guide à la politique américaine. 
3 Trois phénomènes connexes sont analysés: le caractère insaisissable d'une réforme militaire en profondeur; le rôle politique des généraux et des officiers supérieurs; les « arrangements » institutionnels du contrôle civil sur les forces armées.

Pourquoi n'y eut-t-il pas de réforme significative depuis la chute de l'URSS ? Barany postule que, depuis les années 1980, les élites militaires ont pris une place inacceptable dans la politique russe. Leur influence sur les décisions politiques, considérablement renforcée, s'est traduite essentiellement par une opposition aux réformes, les généraux craignant la réduction du nombre des officiers et des conscrits, donc la disparition d'une force de travail. Mais surtout une telle réforme s'opposerait à la conception toujours persistante d'une possible guerre de grande ampleur avec les USA et l'OTAN nécessitant la mobilisation de millions d'hommes.

5 La présidence de Gorbačev, puis celle de El'cin, aurait permis aux militaires d'accéder à un rôle politique (en participant aux élections, en critiquant publiquement le pouvoir), en rupture avec l'époque soviétique où le rôle politique de l'armée était limité. Avec Putin, cette tendance est moindre, mais le président, par la nomination de militaires à des postes-clés du pouvoir, a favorisé l'opposition aux réformes. La responsabilité de l'absence de réforme majeure et de la présence croissante en politique des militaires incombe au président, auteur du déclin de l'importance du législatif et de l'exécutif dont l'équilibre garantit dans les démocraties occidentales le contrôle civil sur l'armée.

6 Pour le démontrer, Barany recourt au cadre théorique de l'approche institutionnelle. Le concept de « déclin institutionnel »- « processus marqué par l'érosion et l'effondrement des règles acceptées et observées ainsi que des normes gouvernant la conduite organisationnelle » - est associé à celui de path dependence que l'on pourrait traduire par "dépendance au chemin emprunté »: une fois une décision prise, il est difficile de changer de voie. En d'autres termes, les décisions peuvent restreindre les choix à venir. Ce concept focalise l'attention sur des moments « fondateurs " (formative moment) quand la voie empruntée est suivie ou modifiée. Notons d'emblée que ce type d'approche tend à mettre en valeur les rigidités institutionnelles aux dépens de celle qui mettrait l'accent sur les flexibilités. Cette dernière aurait été plus pertinente dans le cas d'un régime russe autoritaire dans la mesure où cette orientation, comme tout porte à le croire, est confirmée depuis 1993, selon Barany lui-même.

7 On peut également regretter que ce questionnement global sur les relations civilomilitaires en Russie ne tienne pas suffisamment compte des stratégies politiques des gouvernants envers la société, qui ne sont ici qu'effleurées. Or ces stratégies viennent brouiller la perception qu'ont les militaires du rôle qu'ils sont appelés à jouer dans un contexte sociétal particulier et la perception que les observateurs de la Russie ont du rôle que les militaires entendent jouer ou jouent effectivement sur la scène intérieure. Telle est sans doute la principale faiblesse d'un livre qui, à vouloir théoriser et se concentrer strictement sur le concept restrictif de "relations civilo-militaires », ne porte pas suffisamment d'attention à la place des forces armées dans la société russe postsoviétique (à ses aspects culturels, sociaux et symboliques notamment), au risque de surinterprétations ou d'affirmations péremptoires.

8 Barany identifie trois moments-clés qui ont marqué les relations civilo-militaires au cours de ces deux dernières décennies, moments qui ont été facteur de renforcement du déclin institutionnel par la présence accrue des militaires en politique. Gorbačev invite les militaires à participer activement au processus politique, El'cin encourage ce mouvement, Putin nomme des généraux à des postes politiques et se montre réticent à 
imposer des réformes aux forces armées. Chacun de ces moments, affirme Barany, a détourné la Russie de la voie démocratique.

Le travail de Barany est fondé sur l'analyse de sources primaires (articles de la presse russe) et de sources secondaires (articles de revues, ouvrages sur les relations civilomilitaires). On peut déplorer qu'il n'ait pas été l'occasion d'entretiens avec les parties concernées.

Ses cinq chapitres sont, à l'origine, des articles déjà publiés, remaniés et complétés.

11 Fort habilement, le premier chapitre est consacré à la crise du Kursk et à sa gestion par Vladimir Putin. Cet épisode est instructif à bien des égards : non seulement, comme l'indique très justement Barany, il est révélateur de la manière dont Putin gère une crise, mais de plus il en dit long tant sur l'état des forces armées que sur le respect de la vie humaine en Russie. Il va marquer une nouvelle ère dans la relation du pouvoir aux médias et à la société dans son ensemble : c'est le signal de la mise en place d'un contrôle sur les médias (avec la publication de la "doctrine de sécurité de l'information" élaborée sous $\mathrm{El}^{\prime}$ cin) et d'une tentative d'uniformisation de l'information diffusée à l'ensemble du pays qui se fait sur la base de structures mises aussi en place par El'cin dans un souci de contrôle de l'information dans les régions motivé par la crainte de tendances centrifuges. L'armée est mise à contribution dans ce processus: elle est appelée à jouer un rôle de censeur et de « brouilleur » d'informations à destination de la société, notamment en provenance de Tchétchénie, mais pas seulement.

Le deuxième chapitre affirme que les forces armées ont pendant vingt ans souffert du déclin institutionnel. Une des manifestations de ce déclin est la faiblesse des crédits. Très bien documenté, l'auteur passe en revue l'évolution des doctrines (avec leurs contradictions internes, le poids et l'impact de l'ancien héritage stratégique et conceptuel dans la Russie contemporaine), mais aussi la dégradation tant matérielle que sociale depuis 1985 des forces armées et de leur performance au combat dans les guerres d'Afghanistan et de Tchétchénie.

Barany soutient que cette dégradation continue des forces armées a provoqué dans le corps des officiers un ressentiment considérable qui a servi de catalyseur à leur engagement politique. C'est l'objet du chapitre III. Au chapitre IV,Barany affirme que le principal frein aux réformes militaires radicales est politique, et non économique ou social : c'est la présence en politique des généraux et leur opposition à une réforme radicale. Le dernier chapitre est consacré à l'étude de deux institutions civiles, la présidence et le pouvoir législatif, et à l'examen de l'influence politique des ministères dits « de force » (ministères de la Défense, de l'Intérieur).

Si la démonstration est convaincante pour Gorbačev et $\mathrm{El}^{\prime}$ cin, elle l'est moins pour la période de Putin. Selon Barany, les généraux sont globalement opposés aux réformes, les placer à des postes politiques clés est le facteur majeur de blocage des réformes. Enfin, Putin craindrait d'imposer une réforme en profondeur à une armée qui est la base de son soutien.

Même si l'on ne peut qu'être d'accord avec Barany sur ce point, l'idée que « les généraux » s'opposent aux réformes et veulent imposer une ligne dure parait simplificatrice. Comment ne pas admettre qu'il y a, au sein de l'armée russe, d'une part des officiers progressistes conscients de la nécessité de réformer l'armée mais aussi des officiers conservateurs qui ont une conscience aiguë des enjeux dans la Russie aujourd'hui. Reste à savoir pourquoi ces généraux n'ont pas choisi de se faire entendre. 

de siloviki). Les polpredy (les représentants plénipotentiaires du président) mentionnés par Barany jouent principalement un rôle de supervision des agences fédérales territoriales et de surveillance des activités des gouverneurs. Mais il est vraisemblable que les messages ambigus de Putin appelant l'armée à jouer un rôle plus affirmé sur la scène intérieure (de contrôle et d'éducation notamment) et son nouveau positionnement international ont donné aux généraux le sentiment qu'ils pouvaient regagner du terrain sur le plan politique ; le débat sur la doctrine leur a donné l'occasion de le faire.

Il semble inexact que la base du soutien de Putin soit l'armée, et que par conséquent le président hésite à imposer des réformes aux généraux. Putin n'a pas besoin aujourd'hui de l'appui de l'armée pour rester au pouvoir. Il a gagné à sa cause la majorité du pays, même si à l'évidence le soutien des militaires, qui se sentent culturellement proches de lui, est indéniable. Il n'a pas reculé devant des décisions que certains généraux n'approuvaient pas, comme la coopération avec les États-Unis dans le cadre de la guerre en Afghanistan, ou encore l'installation des bases américaines en Asie centrale. Il n'a pas craint non plus de réduire l'autonomie de l'armée acquise à la faveur de la première guerre en Tchétchénie, lui retirant la gestion de la crise au profit du FSB puis du MVD pendant la seconde, rétablissant la subordination de l'état-major au ministère de la Défense en 2004. Enfin, il n'a pas hésité à apporter une dernière touche à la réforme administrative du ministère de la Défense, nommant à la tête du Bureau de l'information (domaine sensible touchant au contrôle de la société auquel l'armée a été pleinement associée par le président) un ancien haut gradé du KGB, rappelant ainsi à l'armée les limites de son autonomie.

C'est un livre synthétique et clair que nous offre Zoltan Barany, réflexion stimulante sur les relations civilo-militaires. Il intéressera tout autant les étudiants et chercheurs du domaine qu'un public plus large concerné par le développement de la société russe près de vingt ans après la disparition de l'Union soviétique. 